ICAASET-2021, 20-21 May, 2021, K.R. Mangalam University, Gurugram

International Journal of Technical Research \& Science (Special Issue) ISSN No.:2454-2024 (online)

\title{
RESOURCE ALLOCATION FOR DEVICE- TO-DEVICE COMMUNICATION IN THREE-TIER NETWORK USING STACKELBERG GAME THEORY
}

\author{
Roopsi Rathi ${ }^{1}$, Neeraj Gupta ${ }^{2}$ \\ E-Mail Id: ratheeroopsi@gmail.com, neeraj.gupta@krmangalam.edu.in \\ K.R. Mangalam University, Gurgaon, Haryana
}

\begin{abstract}
Device-to-Device (D2D) underlay cellular networks can significantly improves the user's throughput. Nevertheless, the three-tier interference between macrocell- femtocell- D2D systems is becoming challenging in the heterogeneous network. In this paper, first we formulated a Stackelberg game approach, with multiple leadersmultiple followers, macrocell base station (M-BS), and femtocell base station (F-BS) as the leaders and D2D pairs as followers. The leaders minimize the interference by charging prices from followers and followers react to this charging price and find an optimal transmit power. In current work D2D pairs are categorized into sub-classes based on their movement inside the network, each class represented to a different utility function. From different utility functions we get the optimal price for leaders and optimal transmit power for followers. The simulation results show that the proposed approach reduces the transmit power according to the $\mathrm{D} 2 \mathrm{D}$ users' requirement and improves system throughput.
\end{abstract}

Keyword: Resource allocation, Stackelberg game, Price, Transmit power.

\section{INTRODUCTION}

In future generations, it is predictable that the number of devices/users and user's demand will upsurge significantly. Device-to-Device (D2D) communication aims to help to reduce the traffic load off on the cellular network. D2D communication allows direct transmission between users (which is located in close proximity) without the involvement of the base station (central network operator) [1]. D2D users have two transmission modes, either licensed (i.e. inband) or unlicensed (i.e. outband) spectrum in D2D communication. Inband mode signifies that D2D users share cellular spectrum, whereas in outband mode D2D users have been allocated dedicated spectrum resources [2]. D2D communication underlay cellular network offers various benefits, such as system capacity, reliability, reduced latency, improved spectral efficiency, and throughput for cellular and D2D users in the network[3][4]. Nonetheless, D2D communication faces various challenges such as resource allocation, interference management, mode selection, QoS, and power control [5].

In D2D communication, D2D pairs share the same resource blocks of the cellular users, which causes excessive intra-cell and co-channel interference to cellular users and D2D users reusing the same resources [6][7]. Hence, resource allocation and interference management problem is vital in D2D communication. Also, it is a challenging task to enhance the overall performance of the heterogeneous (macro and femtocell) networks, as the receiver suffers three-tier interference, i.e. macro cell to device, femtocell to device and device to device interference within the system[8][9]. To coordinate and avoid interference between users, it is necessary to take an appropriate resource allocation and the interference management approach in the network as in [10].

In the literature survey, the Game theory is used as a tool for the resource allocation and interference management aspects of D2D communication. It facilitates the decision-making process that interacts between players as cellular and D2D users competing for resources. A game involves a set of rational players, where each player selects an action from a strategy set to maximize their payoff (utility) function with regard to all other players [11]. In a noncooperative power control games [6][10][12] minimize the transmit power of users in D2D communication. In [13] the author proposed a combinatorial auction-based approach where cellular user' resources are assigned to multiple D2D users by an iterative bid for maximizes revenue for the auctioneer. In [8][14] the authors proposed joint scheduling and resource allocation approach where a Stackelberg game theory model was applied. The macrocell user and femtocell users act as leaders and D2D users are the followers. It was shown that system model achieves better performance rate for D2D users and as well as for cellular users in heterogeneous networks. Stackelberg game set up the hierarchical structure, it has a leader, and follower concept, where leader acts first and follower observes the leader's strategies then decide its scheme. The leaders demand some additional amount as fees from the followers for utilizing the channel. Then evaluate the optimal price and optimal power for leaders and followers respectively. As in [15], different utility functions are designed for followers, which are suitable to provide particular communication services as per their requirements. The primary objective is to reduce transmit power per D2D pair and guarantee the quality of experience.

Prior work has assumed a static environment where users are stable in the D2D communication network. At the same time, D2D users could be fixed and dynamic in place within the network in the real-time scenario. This paper mainly focuses on the multiple leaders- multiple follower's framework of the Stackelberg game theory. The followers' multiple utility functions are assigned based on the classification of the D2D users, and the categorization of D2D users depends on the behavior or movement of the users. Section 2 is dedicated to system model. Section 3 deals with the problem formulation utilizing the multi-level Stackelberg game. Simulation results for the proposed scheme are presented in section 4. Finally, section 5 concludes the work. 
ICAASET-2021, 20-21 May, 2021, K.R. Mangalam University, Gurugram

International Journal of Technical Research \& Science (Special Issue) ISSN No.:2454-2024 (online)

\section{SYSTEM MODEL}

The system model considers the uplink resource sharing, which includes an M-BS, F-BS, and D2D pairs system in a single cell. M-BS is situated at the center of the cell along with cellular user equipment (CUE). And femtocell randomly located along with F-BS inside the same cell with femtocell user equipment (FUE). The D2D pairs consist of a transmitter and a receiver. D2D pairs are organized into three subclasses as stable class, internal dynamic class and external dynamic class. In the stable class, D2D pairs are allocated within the femtocell range in a constant position. In the internal dynamic class, D2D pairs are allocated within the femtocell range with their movement allowed inside the cell. In the external dynamic class, D2D pairs are allocated within the macrocell range outside the femtocell range. Here, in the current work, we presume a fixed number of CUEs, E, and orthogonal channels, are assigned to the CUES by M-BS. Non-orthogonal channels are assigned to D D2D pairs by M-BS. In other words CUE's channels are shared by D D2D pairs and femtocell system. The number of D2D pairs are more than CUEs.

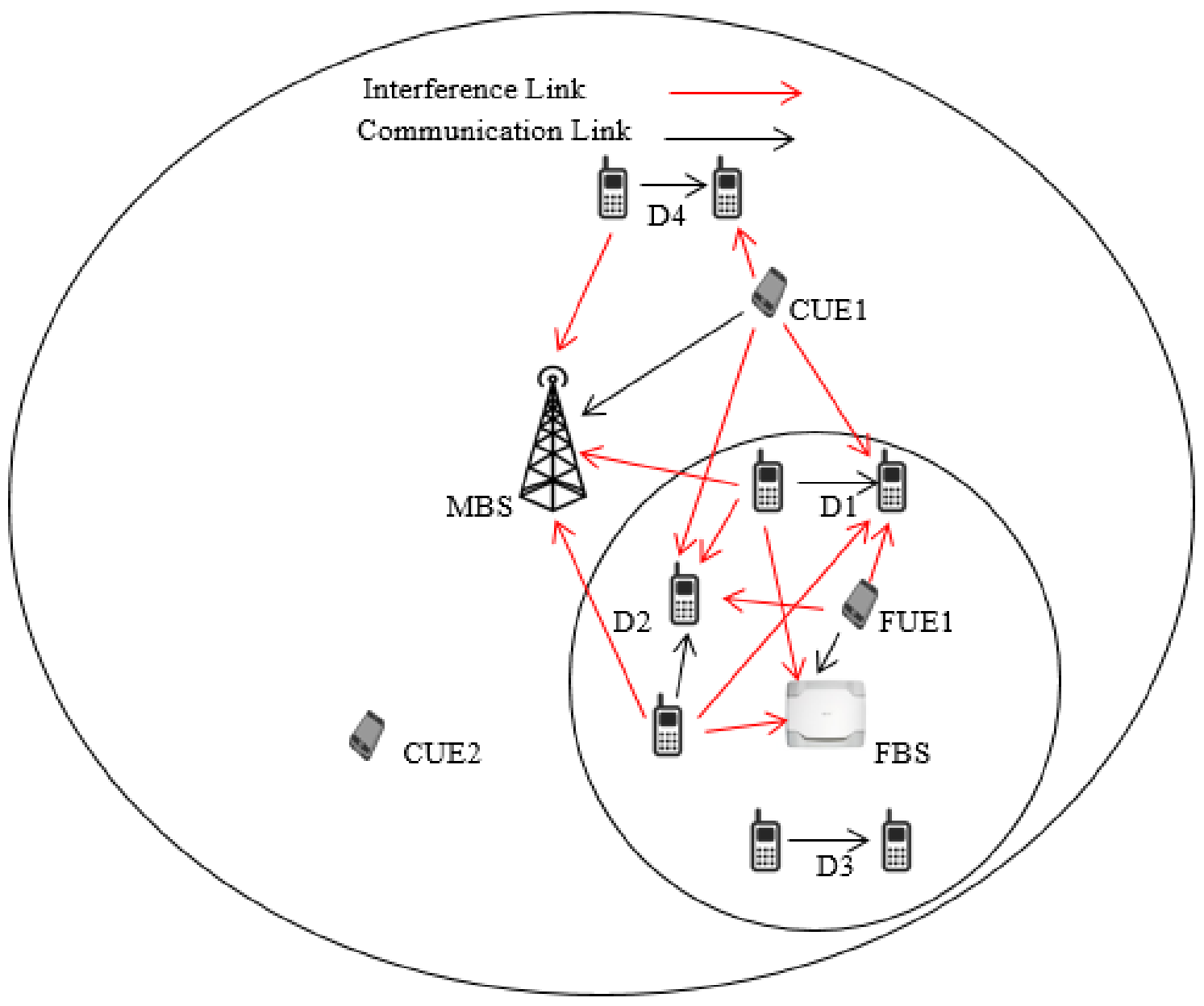

Fig. 2.1 System Model

As shown in fig. 2.1, CUE and FUE $e$ from the set of E and D2D pair i from the set D $(e \in E, i \in D)$ randomly allocated out of and within the femtocell range, respectively. D1 and D2 receivers suffer inter-cell and co-channel interference. M-BS and F-BS suffer interference from D1, D2 receivers, CUE1 and FUE1.

The received SINR at M-BS:

$$
\gamma_{M}=\frac{G_{B}^{M} P_{e}^{1}}{\sum_{h \in H} G_{M}^{h} P_{d}^{h}}+\frac{1}{N_{0}}
$$

The received SINR at F-BS:

$$
\gamma_{F}=\frac{G_{B}^{F} P_{e}^{2}}{\sum_{h \in H} G_{F}^{h} P_{d}^{h}}+\frac{1}{N_{0}}
$$

The received SINR at D2D pairs:

$$
\gamma_{D}=\frac{G_{d}^{i} P_{d}^{i}}{\sum_{e} G_{M}^{i} P_{e}^{1}+\sum_{e} G_{F}^{i} P_{e}^{2}+\sum_{h \in H, h \neq i} G_{i}^{h} P_{h}^{d}}+\frac{1}{N_{0}}
$$

Where, $G_{B}^{M}$ gain between $\mathrm{e}^{\text {th }}$ CUE and M-BS, $G_{B}^{F}$ gain between $\mathrm{e}^{\text {th }}$ FUE and F-BS, $P_{e}^{1}$ and $P_{e}^{2}$ transmit power of $\mathrm{e}^{\text {th }}$ CUE and $\mathrm{e}^{\text {th }}$ FUE, $G_{M}^{h}$ gain between H D2D pair and M-BS, $G_{F}^{h}$ gain between H D2D pair and F-BS, $G_{d}^{i}$ gain between $\mathrm{i}^{\text {th }} \mathrm{D} 2 \mathrm{D}$ transmitter and receiver, $P_{d}^{i}$ transmit power of $\mathrm{i}^{\text {th }} \mathrm{D} 2 \mathrm{D}$ pair, $G_{M}^{i}$ gain between $\mathrm{i}^{\text {th }} \mathrm{D} 2 \mathrm{D}$ receiver and $\mathrm{e}^{\text {th }}$ CUE, $G_{F}^{i}$ gain between $\mathrm{i}^{\text {th }} \mathrm{D} 2 \mathrm{D}$ receiver and $\mathrm{e}^{\text {th }}$ FUE, $G_{i}^{h}$ gain between H D2D pairs and $\mathrm{i}^{\text {th }} \mathrm{D} 2 \mathrm{D}$ receiver, $P_{h}^{d}$ interfering transmit power of D2D pair and $N_{0}$ noise power. 
ICAASET-2021, 20-21 May, 2021, K.R. Mangalam University, Gurugram

International Journal of Technical Research \& Science (Special Issue) ISSN No.:2454-2024 (online)

\section{STACKELBERG GAME THEORY}

D2D communication underlay heterogeneous networks primarily focus on allocating resources and the power control process of the D2D users. The D2D communication can use the proximity between users for better throughput performance of the system. At the same time, interference from D2D pairs to macrocell or femtocell network should be limited. Consequently, the transmit power of D2D users should be appropriately organized. D2D users will transmit with maximum power to maximize their utility, while cellular users will indicate not to share the channel with D2D users. This is an incompetent solution because the D2D users cannot get access to the network. We proposed a Stackelberg game approach with different utility functions for leaders and followers. The objective of the proposed method to coordinate D2D network- to- macrocell/femtocell network interference and allocation of resources within the system network efficiently whereas enhancing the throughput of D2D users. Firstly, this section frames the utility functions of multiple leaders and followers and then shows the optimization problem for leaders and followers.

\subsection{Utility Function}

Based on the above analysis, mainly concentrate on multiple leaders and followers framework. M-BS and F-BS act as leaders and D2D pairs act as followers. Here, leaders (M-BS and F-BS) demand channel fees from followers (D2D pairs) for reusing their channel resources. By giving a channel fee D2D users can decide to transmit so to maximize their profit. The utility of the leaders can be illustrated as their gain and add the channel fee which earns from the followers.

D2D pairs are categorized into sub-classes in the current work, i.e. static class, internal dynamic class, and external dynamic class. This classification is based on their stable or dynamic behavior of users. In static class, D2D users are in a constant position within the femtocell area. The D2D pairs which relate to this class require less transmit power than other sub-classes. In the internal dynamic class, D2D users are roaming within the femtocell area. Its utility function is similar to a static class, but it differs based on velocity. At every TTI (transmission time interval) D2D pair's position is changed. Here, D2D pairs require more transmit power than the static class. In the external dynamic class, D2D pairs are in a dynamic position out of the femtocell area. It requires more transmit power than other classes. Mathematically, M-BS, F-BS, and D2D pair's utility functions can be described, respectively as:

$$
\begin{aligned}
& U_{B S}^{M}=\log _{2}\left(1+\frac{G_{B}^{M} P_{e}^{1}}{G_{M}^{h} P_{d}^{h}+N_{0}}\right)+P_{1}^{i} G_{M}^{i} \\
& U_{B S}^{F}=\log _{2}\left(1+\frac{G_{B}^{F} P_{e}^{2}}{G_{F}^{h} P_{d}^{h}+N_{0}}\right)+P_{2}^{i} G_{F}^{i} \\
& U_{D 2 D}^{S}=\log _{2}\left(1+\frac{G_{d}^{i} P_{d}^{i}}{G_{M}^{i} P_{e}^{1}+G_{F}^{i} P_{e}^{2}+G_{i}^{h} P_{h}^{d}+N_{0}}\right)-P_{1}^{i} G_{M}^{i}-P_{2}^{i} G_{F}^{i} \\
& U_{D 2 D}^{D}=\log _{2}\left(1+\frac{G_{d}^{i} P_{d}^{i}}{G_{M}^{i} P_{e}^{1}+G_{i}^{h} P_{h}^{d}+N_{0}}\right)-P_{1}^{i} G_{M}^{i}
\end{aligned}
$$

Where $U_{B S}^{M}, U_{B S}^{F}, U_{D 2 D}^{S}$ and $U_{D 2 D}^{D}$ defines the utility functions of M-BS, F-BS, static- internal dynamic class, and external dynamic class, respectively. $P_{1}^{i}$ and $P_{2}^{i}$ show channel fees of M-BS and F-BS, respectively..

\subsection{Optimization Problem}

The leaders and followers have optimization problems to determine a set of channel fees and proper transmit power, which maximizes their utility

$$
\begin{gathered}
\max U_{B S}^{M}, U_{B S}^{F} \\
\text { s.t. } P_{1}^{i}>0, P_{2}^{i}>0 \\
\max U_{D 2 D}^{S}, U_{D 2 D}^{D} \\
\text { s.t. } p_{\min } \leq P_{d}^{i} \leq p_{\max }
\end{gathered}
$$

\section{SIMULATION RESULTS}

The various simulations are executed in evaluating the performance of the proposed approach. We considered two BSs such as M-BS $500 \mathrm{~m}$ radius and F-BS $50 \mathrm{~m}$ radius. The CUEs, FUEs, and D2D pairs are consistently distributed within the cell. We analyze the D2D network, where two D2D UEs in a D2D pair are close enough to satisfy the maximum D2D transmission distance. We assume that each CUE transmits at $23 \mathrm{dBm}$ range i.e. maximum transmission power. Table 1 defines the simulation parameters. Initially, $e$ CUE and FUE assigned to $h$ D2D pairs, where $h \in H, h \neq i$ and a set $H \subset D$ and $e \in E$, to reuse their channels. Moreover, $h$ D2D pairs are equally assigned to each D2D sub-class.

Table-4.1 Simulation parameters and its values

\begin{tabular}{|l|l|}
\hline Parameter & Value \\
\hline Macrocell radius & $500 \mathrm{~m}$ \\
\hline Femtocell radius & $50 \mathrm{~m}$ \\
\hline Number of the CUE & 3 \\
\hline Number of the FUE & 3 \\
\hline Number of D2D pairs & 6 \\
\hline
\end{tabular}


ICAASET-2021, 20-21 May, 2021, K.R. Mangalam University, Gurugram

International Journal of Technical Research \& Science (Special Issue) ISSN No.:2454-2024 (online)

\begin{tabular}{|l|l|}
\hline Distance between D2D transmitter and receiver & $10 \mathrm{~m}$ \\
\hline Range of D2D transmit power & $0-23 \mathrm{dBm}$ \\
\hline Bandwidth & $180 \mathrm{kHz}$ \\
\hline Transmission time interval & $1 \mathrm{~ms}$ \\
\hline
\end{tabular}

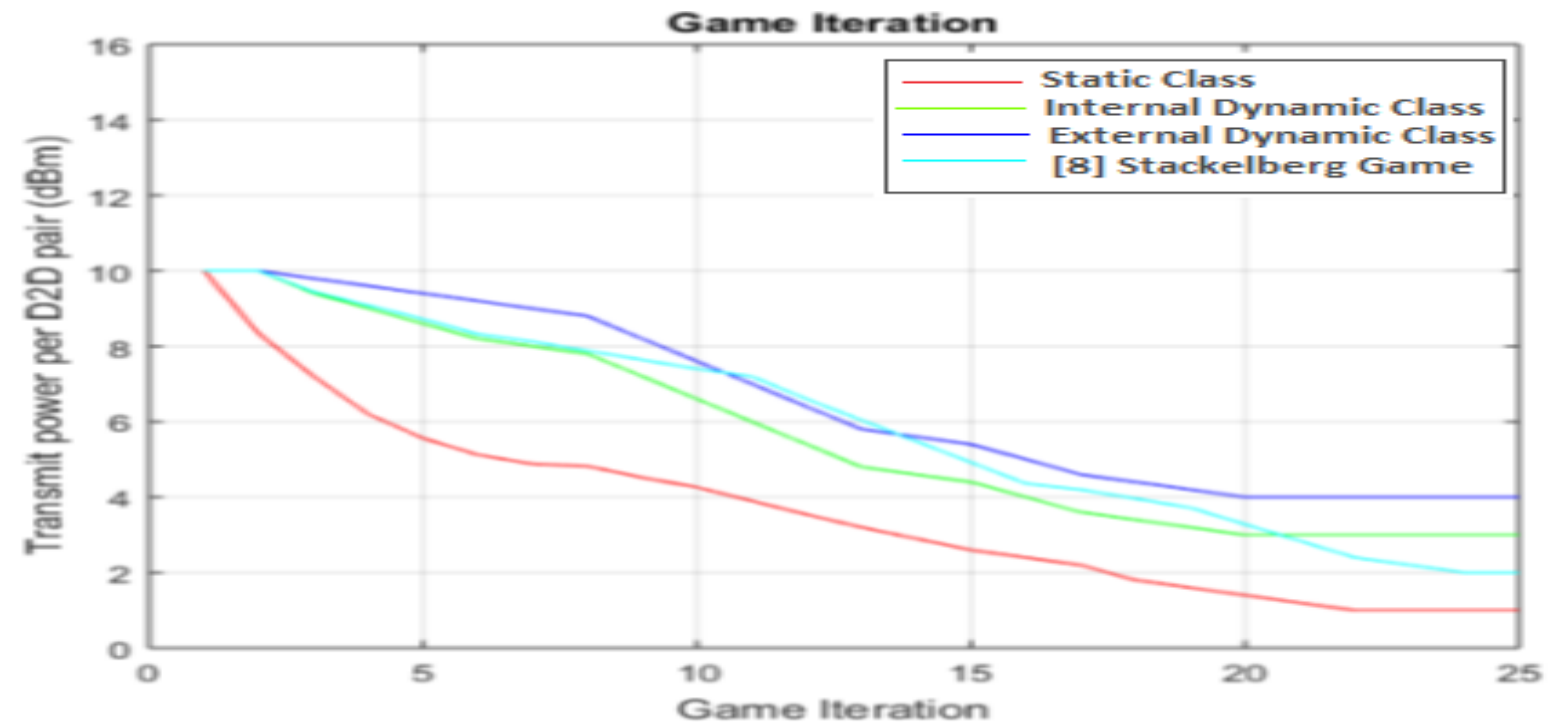

Fig. 2.2 Transmit Power per D2D pair

Fig. 2.2 shows the transmission power per D2D pair with game iteration for the network. It can be seen that that D2D pair's transmit power reduce according to the classes. Static class D2D pairs require less transmit power than the other two classes. Internal dynamic class D2D pairs require less transmit power than external dynamic class D2D pairs. It can be observed that our proposed static class requires less transmit power as compared to [8].

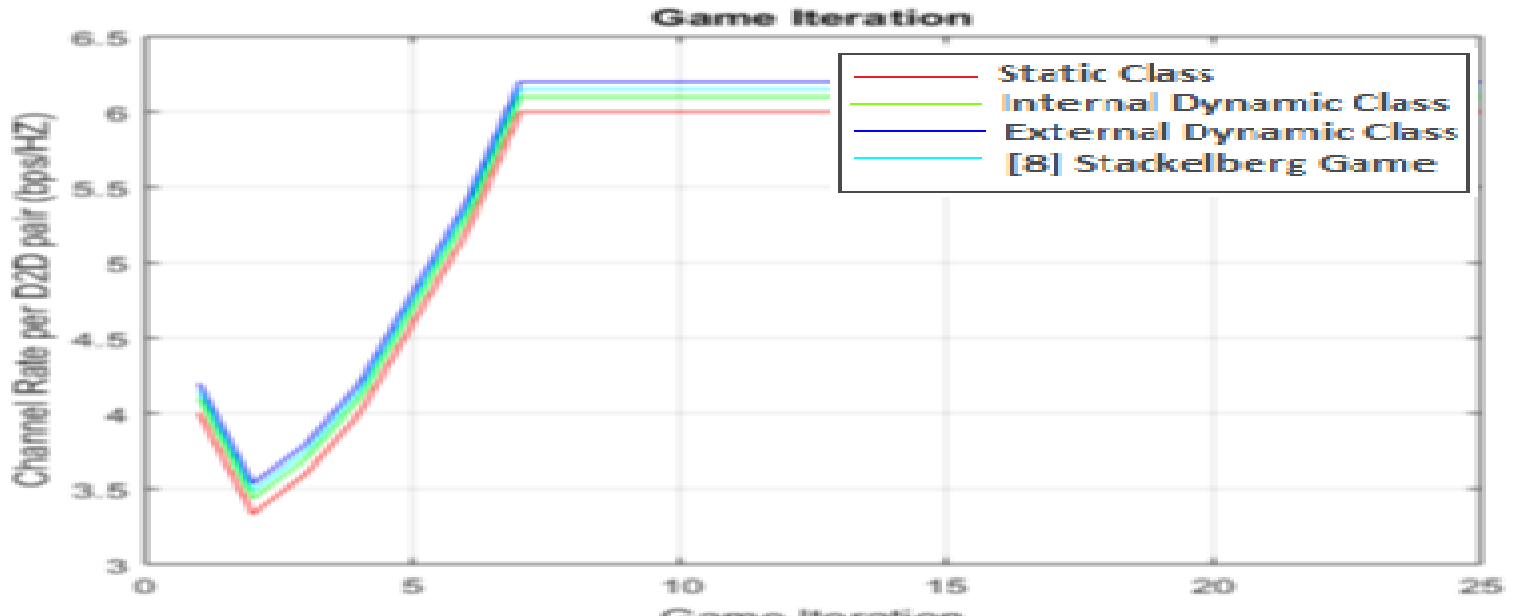

Fig. 2.3 Channel rate per D2D pair

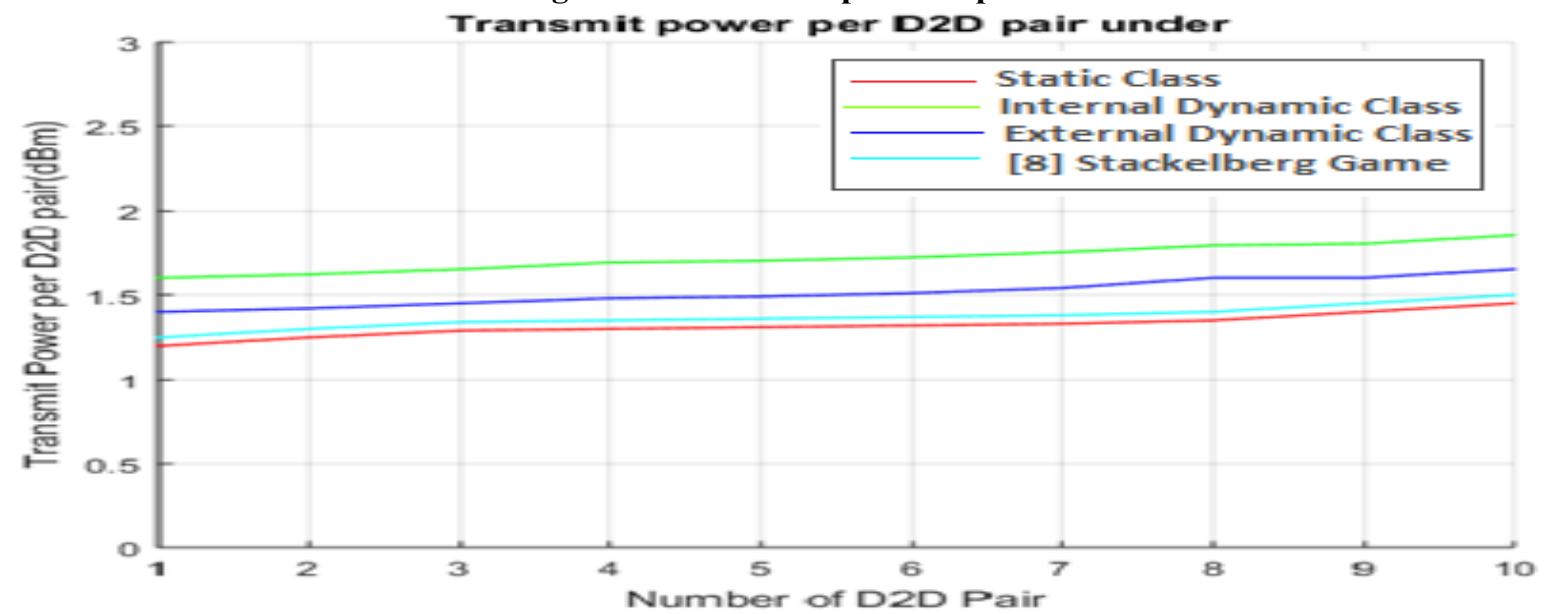

Fig. 2.4 Transmit power with number of D2D pairs

Fig. 2.3 shows the channel rate per D2D pair with game iteration. We observed that each subclass of D2D pair achieves a better channel rate per DD pair. The joint scheduling [8] performs a better channel rate than our 
ICAASET-2021, 20-21 May, 2021, K.R. Mangalam University, Gurugram

International Journal of Technical Research \& Science (Special Issue) ISSN No.:2454-2024 (online) proposed approach, but if we consider the results of fig. 2, [8] requires more transmit power which occurs more inter-cell interference than the proposed approach. Fig. 2.4, shows transmit power with a number of D2D pairs. As the number of D2D pair increases more transmit power is required. This is because as the number of D2D pairs reuses the CUEs channels and it occurs interference within the network.

\section{CONCLUSION}

This paper proposes a Stackelberg game approach with multiple utility functions for leaders and followers to allow efficient power control and resource allocation of D2D communication in the heterogeneous network. The followers are classified into three classes based on their movement and the cell region. Based on the multiple utility functions, the appropriate price for reusing the channels and transmit power of each class of D2D pairs is adjusted in order to enhance the performance of the system. The aim is to minimize transmit power per D2D pair and achieve a better UE rate for CUEs and D2D users. Simulation results have shown that the proposed approach can reduce the transmission power and improve channel rate per $\mathrm{D} 2 \mathrm{D}$ pair.

\section{REFERENCES}

[1] Tehrani, M. N., Uysal, M., Yanikomeroglu, H. (2014): Device-to-device communication in 5G cellular networks: challenges, solutions, and future directions. IEEE Communications Magazine, 52(5), 86-92.

[2] Mach, P., Becvar, Z., Vanek, T. (2015): In-band device-to-device communication in OFDMA cellular networks: A survey and challenges. IEEE Communications Surveys \& Tutorials, 17(4), 1885-1922.

[3] Jameel, F., Hamid, Z., Jabeen, F., Zeadally, S., Javed, M. A. (2018): A survey of device-to-device communications: Research issues and challenges. IEEE Communications Surveys \& Tutorials, 20(3), 21332168.

[4] Feng, D., Lu, L., Yuan-Wu, Y., Li, G. Y., Feng, G., Li, S. (2013): Device-to-device communications underlaying cellular networks. IEEE Transactions on communications, 61(8), 3541-3551.

[5] Liu, J., Kato, N., Ma, J., Kadowaki, N. (2014): Device-to-device communication in LTE-advanced networks: A survey. IEEE Communications Surveys \& Tutorials, 17(4), 1923-1940.

[6] Song, L., Niyato, D., Han, Z., Hossain, E. (2014): Game-theoretic resource allocation methods for deviceto-device communication. IEEE Wireless Communications, 21(3), 136-144.

[7] Wang, F., Xu, C., Song, L., Han, Z. (2014): Energy-efficient resource allocation for device-to-device underlay communication. IEEE Transactions on Wireless Communications, 14(4), 2082-2092.

[8] He, Y., Wang, F., Wu, J. (2014): Resource management for device-to-device communications in heterogeneous networks using stackelberg game. International Journal of Antennas and Propagation, 2014.

[9] Han, Y., Tao, X., Zhang, X. (2018, April): Power allocation for device-to-device underlay communication with femtocell using stackelberg game. In 2018 IEEE Wireless Communications and Networking Conference (WCNC) (pp. 1-6). IEEE.

[10] Phunchongharn, P., Hossain, E., Kim, D. I. (2013): Resource allocation for device-to-device communications underlaying LTE-advanced networks. IEEE Wireless Communications, 20(4), 91-100.

[11] Han, Z., Niyato, D., Saad, W., Başar, T., Hjørungnes, A. (2012): Game theory in wireless and communication networks: theory, models, and applications. Cambridge university press.

[12] Fodor, G., Della Penda, D., Belleschi, M., Johansson, M., Abrardo, A. (2013, June): A comparative study of power control approaches for device-to-device communications. In 2013 IEEE International Conference on Communications (ICC) (pp. 6008-6013). IEEE.

[13] Xu, C., Song, L., Han, Z., Li, D., Jiao, B. (2012, December): Resource allocation using a reverse iterative combinatorial auction for device-to-device underlay cellular networks. In 2012 IEEE Global Communications Conference (GLOBECOM) (pp. 4542-4547). IEEE.

[14] Wang, F., Song, L., Han, Z., Zhao, Q.,: Wang, X. (2013, April): Joint scheduling and resource allocation for device-to-device underlay communication. In 2013 IEEE wireless communications and networking conference (WCNC) (pp. 134-139). IEEE.

[15] Sawyer, N., Smith, D. B. (2018): Flexible resource allocation in device-to-device communications using stackelberg game theory. IEEE Transactions on Communications, 67(1), 653-667. 